\title{
Robust Linear Longitudinal Feedback Control of a Flapping Wing Micro Air Vehicle
}

\author{
Lidia Mar'ı Belmonte, R. Morales, Antonio Fern'andez-Caballero, and \\ Jos'e A. Somolinos
}

\begin{abstract}
This paper falls under the idea of introducing biomimetic miniature air vehicles in ambient assisted living and home health applications. The concepts of active disturbance rejection control and flatness based control are used in this paper for the trajectory tracking tasks in the flapping-wing miniature air vehicle (FWMAV) time-averaged model. The generalized proportional integral (GPI) observers are used to obtain accurate estimations of the flat output associated phase variables and of the time-varying disturbance signals. This information is used in the proposed feedback controller in (a) approximate, yet close, cancelations, as lumped unstructured time-varying terms, of the influence of the highly coupled nonlinearities and (b) the devising of proper linear output feedback control laws based on the approximate estimates of the string of phase variables associated with the flat outputs simultaneously provided by the disturbance observers. Numerical simulations are provided to illustrate the effectiveness of the proposed approach.
\end{abstract}

\section{Introduction}

The creation of flapping wing micro air vehicles (FWMAV) is a challenging problem. The potential benefits for insect-like flapping wing micro air vehicles are numerous [1]. The hovering ability of insects, coupled with the ability for a quick transition to forward flight, provide an ideal indoor/outdoor reconnaissance platform for search and rescue, reconnaissance and surveillance and ambient assisted living and home health, among others [2]-[8]. Indeed, this paper falls within a project called "Improvement of the Elderly Quality of Life and Care through Smart Emotion Regulation". The long-term objective of the project is to find solutions for improving the quality of life and care of ageing adults at home by using emotion detection and regulation techniques. We believe that miniature air vehicles at home settings are capable of including some sensors that capture the mood of the ageing adults.

Different control methods have been found in the literature. Deng et al. developed in [9] a nominal state-space linear time-invariant model in hover through 


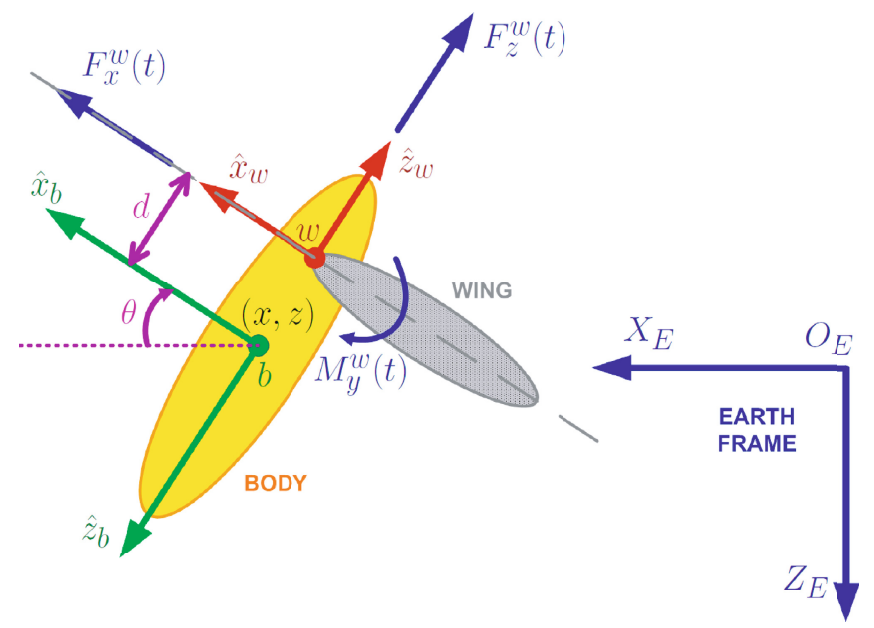

Fig. 1. Coordinate systems and longitudinal motion of FWMAV with respect to the earth frame

linear estimation. Also, a LQG controller was designed and compared with a PD controller. A state feedback attitude controller control scheme using the sensor output as feedback was designed by Schenato et al. [10]. Campolo et al. realized in [11] a geometric approach to robust attitude estimation, derived from multiple and possibly redundant bio-inspired navigation sensors, for attitude stabilization of a micromechanical flying insect.

The use of time-averaging theory has been used within the control of FWMAV because it helps to simplify the complex aerodynamics associated to the flapping wings [12]-[13] because the aerodynamic forces and torques, generated by the wings, affect the behavior of the FWMAV only by their mean values since the dynamics of the body are much slower than the flapping wings ones. Deng et al. provided a methodology to approximate the time-varying body dynamics caused by the aerodynamic forces with time-invariant dynamics using averaging theory and a biomimetic parametrization of wing trajectories [14]. Also, a Linear Quadratic Gaussian (LQG) controller was designed which does not require the knowledge of an accurate model for the insect morphological parameters, such as moment of inertia and mechanical part's sizes, nor an accurate model of the aerodynamics. Rifaï et al. developed in [15] a bounded state feedback control of the forces and torques and takes into account the saturation of the actuators driving the flapping wings and Khan et al. realized in [16] a differential flatness based non-linear controller based on the time-averaging theory for the control of the longitudinal dynamics of FWMAV.

Taking into consideration the highly nonlinear nature of the FWMAV, active disturbance rejection control (ADRC) appears as an excellent methodology for the control of uncertain linear and nonlinear systems (see the work of Han [17] for the initial theoretical aspects of this new area of research). The objective of ADRC stems in the accurate estimation of the unknown part of the controlled system dy- 
namics and proceed to cancel its effects in the feedback control law. Gao and its coworkers have proposed new advances in controllers, including practical applications, in a similar manner to that of Han [18], [19]. On the other hand, Sira-Ramírez and its coworkers have contribute to the area emphasizing the use of generalized proportional integral (GPI) observers [20]-[22].

In this line of action, in this article, we propose a robust observer-based linear output feedback control scheme for the trajectory tracking tasks in the flappingwing miniature air vehicle time-averaged model. The linear observer-based controller design approach rests on using highly simplified models of the inputs differential parameterizations, provided by the flatness property. Within the simplification task proposed, only the order of integration of the subsystems and the control inputs, along with their associated matrix gains, are retained in full detail. All the additive nonlinearities, including their state couplings and complexities, are regarded as, unstructured, time-varying signals that need to be online estimated, and canceled, at the controller specification within an Active Disturbance Rejection Control Scheme. After input gain matrix cancelation, the resulting system consists of pure integration (linear) perturbed systems with time-varying additive disturbances. A set of linear extended observers, here denominated as GPI observers, are capable of accurate on-line estimations of: (1) the output related phase variables; (2) the, state dependent, additive perturbation input signal itself; and (3) the estimation of a certain number of the perturbation input time derivatives. This last feature facilitates the task of perturbation input prediction as GPI observers are the most naturally applicable to the control of perturbed differentially flat nonlinear systems [23]-[25].

The remainder of the article is structured as follows: Section 2 presents the flappingwing miniature air vehicle time-averaged model and its flatness property. Additionally, this section proposes a simplified model of the system and formulates the problem to be solved. Section 3 describes the active disturbance rejection controller design and the results are applied for the stabilization and trajectory tracking problem of the time-averaged model for the flapping-wing miniature air vehicle. Section 4 presents the obtained simulation results and, finally, Section 5 is devoted for the conclusions of this study and future works.

\section{Problem Formulation and Its Flatness Property}

\subsection{System Dynamics}

Consider the following time-averaged model for the flapping-wing miniature air vehicle (FWMAV) based on Newtonian approach derived in [16]:

$$
\begin{aligned}
\dot{x} & =v_{x} C_{\theta}+v_{z} S_{\theta} \\
\dot{z} & =-v_{x} S_{\theta}+v_{z} C_{\theta} \\
\dot{\theta} & =\omega \\
\dot{v}_{x} & =-g S_{\theta}-\omega v_{z}+F_{x} \\
\dot{v}_{z} & =-g C_{\theta}+\omega v_{x}-F_{z} \\
\dot{\omega} & =-\frac{F_{x}}{E}
\end{aligned}
$$


where $S_{\theta}=\sin \theta, C_{\theta}=\cos \theta, g$ is the gravity acceleration, $(x, z)$ are the coordinates of the center of mass in the earth frame, $\theta$ represents the pitch angle, $\left(v_{x}, v_{z}\right)$ express the velocity of the body of the FWMAV in the body frame and $\omega$ is the angular velocity of the body and $\left(F_{x}, F_{z}\right)$ represent the aerodynamic forces. The constant $E=\frac{I_{b}}{m d}$, being $I_{b}$ the moment of inertia of the body about the $y$ axis of the body frame, $m$ is the mass and $d$ denotes the distance from the axis of oscillation to the center of mass of the body. Fig. 1 shows the coordinate systems and longitudinal motion of the FWMAV with regard to the earth frame.

\subsection{Flatness of the System}

According to the theory of differential flatness [21], a dynamic system, $\dot{\mathbf{x}}=\mathbf{f}(\mathbf{x}, \mathbf{u})$, with $\mathbf{x} \in \mathbb{R}^{n}$ and $\mathbf{u} \in \mathbb{R}^{m}$, is said to be differentially flat if there exist, $m$, differentially independent variables called flat outputs (differentially independent meaning that they are not related by differential equations), which are functions of the state vector and, possibly, of a finite number of time derivatives of the state vector (i.e., derivatives of the inputs may be involved in their definition), such that all system variables (states, inputs, outputs, and functions of these variables) can, in turn, be expressed as functions of the flat outputs and of a finite number of their time derivatives. This parameterization establishes a one-to-one mapping from the states and the inputs to the flat outputs.

The proposed system is differentially flat with flat outputs given by the coordinates of the Huygens center of oscillation [22] given by:

$$
F=x+E S_{\theta}, \quad L=z+E C_{\theta}
$$

Proposition 1. The flapping-wing miniature air vehicle given in (1)-(6) is differentially flat, with flat outputs given by $F$ and $L$, i.e., all system variables in (1)-(6) can be differentially parameterized solely in terms of $F, L$, and a finite number of their time derivatives.

Proof. If the equations given in (7) are differentiated with regard to time, we obtain the first and second derivatives of the flat outputs:

$$
\begin{aligned}
\dot{F} & =\dot{x}+E \omega C_{\theta}=v_{x} C_{\theta}+v_{z} S_{\theta}+E \omega C_{\theta} \\
\dot{L} & =\dot{z}-E \omega S_{\theta}=-v_{x} S_{\theta}+v_{z} C_{\theta}-E \omega S_{\theta} \\
\ddot{F} & =\xi S_{\theta} \\
\ddot{L} & =g+\xi C_{\theta}
\end{aligned}
$$

where $\xi=-\left(F_{z}-E \omega^{2}\right)$ is defined as a new virtual input vector. Upon operating with (10) and (11) we achieve:

$$
\begin{gathered}
\xi=\sqrt{\ddot{F}^{2}+(\ddot{L}-g)^{2}} ; \quad \theta=\arctan \left(\frac{\ddot{F}}{\ddot{L}-g}\right) \\
S_{\theta}=\frac{\ddot{F}}{\sqrt{\ddot{F}^{2}+(\ddot{L}-g)^{2}}} ; \quad C_{\theta}=\frac{\ddot{L}-g}{\sqrt{\ddot{F}^{2}+(\ddot{L}-g)^{2}}}
\end{gathered}
$$


If the expressions (10) and (11) are differentiated with regard to time, it is obtained

$$
F^{(3)}=\dot{\xi} S_{\theta}+\xi \omega C_{\theta} ; \quad L^{(3)}=\dot{\xi} C_{\theta}-\xi \omega S_{\theta}
$$

Rearranging terms in (14) yields

$$
\dot{\xi}=\frac{\ddot{F} F^{(3)}+(\ddot{L}-g) L^{(3)}}{\sqrt{\ddot{F}^{2}+(\ddot{L}-g)^{2}}} ; \quad \omega=\dot{\theta}=\frac{F^{(3)}(\ddot{L}-g)-L^{(3)} \ddot{F}}{\ddot{F}^{2}+(\ddot{L}-g)^{2}}
$$

Now, operating with (4) and (5) one obtains

$$
\begin{aligned}
v_{x} & =\dot{x} C_{\theta}-\dot{z} S_{\theta}=\dot{F} C_{\theta}-\dot{L} S_{\theta}-E \omega \\
v_{z} & =\dot{x} S_{\theta}+\dot{z} C_{\theta}=\dot{F} S_{\theta}+\dot{L} C_{\theta}
\end{aligned}
$$

Combining (16) and (17) with (13) and (15), we conclude that $v_{x}$ and $v_{z}$ are also functions of $\left(\dot{F}, \dot{L}, \ddot{F}, \ddot{L}, F^{(3)}, L^{(3)}\right)$. On the other hand, differentiating expressions (14) with regard to time and rearranging terms

$$
\begin{aligned}
F^{(4)} & =S_{\theta} \ddot{\xi}-\frac{\xi C_{\theta}}{E} F_{x}-\omega^{2} \xi S_{\theta}+2 \dot{\xi} \omega C_{\theta} \\
L^{(4)} & =C_{\theta} \ddot{\xi}+\frac{\xi S_{\theta}}{E} F_{x}-\omega^{2} \xi C_{\theta}-2 \dot{\xi} \omega S_{\theta}
\end{aligned}
$$

Similarly, upon operating with (18), it is achieved

$$
\begin{aligned}
\ddot{\xi} & =S_{\theta} F^{(4)}+C_{\theta} L^{(4)}+\omega^{2} \xi \\
F_{x} & =\frac{-E C_{\theta}}{\xi} F^{(4)}+\frac{E S_{\theta}}{\xi} L^{(4)}+2 \frac{E \omega \dot{\xi}}{\xi}
\end{aligned}
$$

Finally, substituting (12), (13) and (15) into (20) shows that all the system variables can be expressed as a function of $(F, L)$ and their derivatives, proving that the flat output vector composed by $(F, L)$ constitute a flat output vector for system (1)-(6).

\subsection{Simplified Model and Problem Formulation}

On the basis of (20), we adopt the following simplified perturbed model for the underlying FWMAV (18):

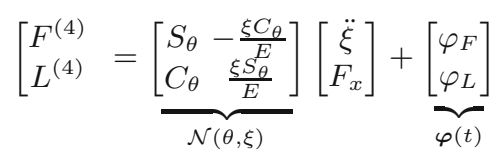

where $\varphi(t)=\left[\varphi_{F}, \varphi_{L}\right]^{T}$ involves state dependent expressions, the possibly unmodeled dynamics and external unknown disturbances affecting the system. We lump 
all these uncertain terms into an unknown but uniformly absolutely bounded disturbance input that needs to be on-line estimated by means of an observer and, subsequently, canceled from the simplified system dynamics via feedback in order to regulate the flat output vector, $[F, L]^{T}$, towards the desired reference trajectories $\left[F^{*}, L^{*}\right]^{T}$. Finally, the formulation of the problem is stated as follows: Given a desired flat output vector of reference trajectories $\left[F^{*}, L^{*}\right]^{T}$, devise a linear multi-input output feedback controller for system (22) such that the flat output vector $[F, L]^{T}$ is forced to track the given reference flat output vector $\left[F^{*}, L^{*}\right]^{T}$. This objective must be achieved even in the presence of unknown disturbances and coupling nonlinearities, represented by $\left[\varphi_{F}, \varphi_{L}\right]^{T}$.

\section{GPI Observer-Based Active Disturbance Rejection Controller}

A GPI observer including a reasonable, self-updating, time-polynomial model is considered for each unknown component disturbance input vector $\varphi(t)$. For this internal model, we use for each component of $\varphi(t)$ an unspecified element of a fifth order family of time-polynomials, denoted by $\varphi_{1}^{(6)}(t)=\left[\varphi_{1 F}^{(6)}, \varphi_{1 L}^{(6)}\right]^{T}=\mathbf{0}$. The GPI observer based flat output feedback controller is devised as follows:

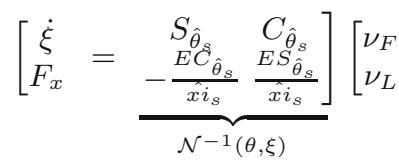

with

$$
\begin{aligned}
& \nu_{F}=-\hat{\varphi}_{1 F s}+\left[F^{*}(t)\right]^{(4)}-\sum_{i=0}^{3} k_{i}^{F}\left(\hat{F}_{s}^{(i)}-\left[F^{*}\right]^{(i)}\right) \\
& \nu_{L}=-\hat{\varphi}_{1 L s}+\left[L^{*}(t)\right]^{(4)}-\sum_{i=0}^{3} k_{i}^{L}\left(\hat{L}_{s}^{(i)}-\left[L^{*}\right]^{(i)}\right)
\end{aligned}
$$

where the quantities with subindex $s$ are smoothing observer variables which are carried out by means of the following clutching function, avoiding possible large peaks in their high gain induced responses:

$$
s_{f}(t)=\left\{\begin{array}{l}
1 \text { for } t>\varepsilon \\
\sin ^{8}\left(\frac{\pi t}{2 \varepsilon}\right) \text { for } t \leq \varepsilon
\end{array}\right.
$$

with $\epsilon=2[s]$. The design coefficients $k_{i}^{F}$ and $k_{i}^{L}, i=0,1,2,3$, are chosen so that the dominant characteristic polynomials are 4 th-degree Hurwitz polynomials, i.e.,

$$
\begin{aligned}
& p_{F}(s)=s^{4}+k_{3}^{F} s^{3}+k_{2}^{F} s^{2}+k_{1}^{F} s+k_{0}^{F} \in \operatorname{Hurwitz}_{4}(s) \\
& p_{L}(s)=s^{4}+k_{3}^{L} s^{3}+k_{2}^{L} s^{2}+k_{1}^{L} s+k_{0}^{L} \in \operatorname{Hurwitz}_{4}(s)
\end{aligned}
$$

render an asymptotically, exponentially convergence of the flat output error vector, $\left[e_{F}, e_{L}\right]^{T}=\left[F-F^{*}, L-L^{*}\right]^{T}$, towards a small vicinity of the origin of the 
tracking error phase space. Furthermore, the variables $\hat{F}^{(j)}=F_{j}$ and $\hat{L}^{(j)}=L_{j}$, $j=0,1, \ldots, 3$ are generated by:

$$
\begin{aligned}
\dot{F}_{0} & =F_{1}+\lambda_{8}^{F}\left(F-F_{0}\right) \\
\dot{F}_{1} & =F_{2}+\lambda_{7}^{F}\left(F-F_{0}\right) \\
\dot{F}_{2} & =F_{3}+\lambda_{6}^{F}\left(F-F_{0}\right) \\
\dot{F}_{3} & =S_{\theta} \ddot{\xi}-\frac{\xi C_{\theta}}{E} F_{x}+\varphi_{1 F}+\lambda_{5}^{F}\left(F-F_{0}\right) \\
\dot{\varphi}_{1 F} & =\varphi_{2 F}+\lambda_{4}^{F}\left(F-F_{0}\right) \\
\dot{\varphi}_{2 F} & =\varphi_{3 F}+\lambda_{3}^{F}\left(F-F_{0}\right) \\
\dot{\varphi}_{3 F} & =\varphi_{4 F}+\lambda_{2}^{F}\left(F-F_{0}\right) \\
\dot{\varphi}_{4 F} & =\varphi_{5 F}+\lambda_{1}^{F}\left(F-F_{0}\right) \\
\dot{\varphi}_{5 F} & =\lambda_{0}^{F}\left(F-F_{0}\right) \\
\dot{L}_{0} & =L_{1}+\lambda_{8}^{L}\left(L-L_{0}\right) \\
\dot{L}_{1} & =L_{2}+\lambda_{7}^{L}\left(L-L_{0}\right) \\
\dot{L}_{2} & =L_{3}+\lambda_{6}^{L}\left(L-L_{0}\right) \\
\dot{L}_{3} & =C_{\theta} \ddot{\xi}+\frac{\xi S_{\theta}}{E} F_{x}+\varphi_{1 L}+\lambda_{5}^{L}\left(L-L_{0}\right) \\
\dot{\varphi}_{1 L} & =\varphi_{2 L}+\lambda_{4}^{L}\left(L-L_{0}\right) \\
\dot{\varphi}_{2 L} & =\varphi_{3 L}+\lambda_{3}^{L}\left(L-L_{0}\right) \\
\dot{\varphi}_{3 L} & =\varphi_{4 L}+\lambda_{2}^{L}\left(L-L_{0}\right) \\
\dot{\varphi}_{4 L} & =\varphi_{5 L}+\lambda_{1}^{L}\left(L-L_{0}\right) \\
\dot{\varphi}_{5 L} & =\lambda_{0}^{L}\left(L-L_{0}\right)
\end{aligned}
$$

where the design coefficients $\lambda_{i}^{F}$ and $\lambda_{i}^{L}, i=0,1, \ldots, 8$, are chosen so that the reconstruction error dynamics dominant characteristic polynomials are 9 th-degree Hurwitz polynomials, i.e.,

$$
\begin{aligned}
& p_{F o}(s)=s^{9}+\lambda_{8}^{F} s^{8}+\lambda_{7}^{F} s^{7}+\ldots+\lambda_{1}^{F} s+\lambda_{0}^{F} \in \operatorname{Hurwitz}_{9}(s) \\
& p_{L o}(s)=s^{9}+\lambda_{8}^{L} s^{8}+\lambda_{7}^{L} s^{7}+\ldots+\lambda_{1}^{L} s+\lambda_{0}^{L} \in \operatorname{Hurwitz}_{9}(s)
\end{aligned}
$$

and their roots are located sufficiently far from the imaginary axis, in the left half of the complex plane, then the trajectories of the flat output estimation error vector, $\left[\tilde{e}_{F}, \tilde{e}_{L}\right]^{T}=\left[F-F_{0}, L-L_{0}\right]^{T}$, and of its time derivatives, will converge to a small neighborhood of the origin of the phase space of the observer estimation error. The further away the roots are located in the left half of the complex plane, the smaller the radius of the disk representing the neighborhood around the origin of the estimation error phase space will be. 


\section{Numerical Simulations}

Numerical simulations were carried out in order to verify the efficiency of the proposed approach in terms of quick convergence of the tracking errors to a small neighborhood of zero and smooth transient responses. The system parameters are: $m=2.5 \cdot 10^{-3}[\mathrm{~kg}]$ and $I_{b}=8.125 \cdot 10^{-7}\left[\mathrm{~kg} \mathrm{~m}^{2}\right]$. The flat output vector $[F, L]^{T}$ has been designed to track the following reference trajectories:

$$
\begin{aligned}
F^{*} & =R \sin (A t)+E \sin (\alpha(t)) \\
L^{*} & =R[\cos (A t)-1]-z_{0}+E \cos (\alpha(t))
\end{aligned}
$$

where $R=7[\mathrm{~m}], z_{0}=0.5[\mathrm{~m}], A=2 \pi / 30[\mathrm{rad} / \mathrm{s}]$ and $\alpha(t)=B_{1} \sin \left(B_{2} t\right)$ being $B_{1}=\pi / 180[\mathrm{rad}]$ and $B_{2}=2 \pi / 30[\mathrm{rad} / \mathrm{s}]$.

The time sampling used in all the simulations is $T=0.001[s]$. The observer gains, $\left\{\lambda_{8}^{F}, \ldots, \lambda_{0}^{F}\right\}$ and $\left\{\lambda_{8}^{L}, \ldots, \lambda_{0}^{L}\right\}$ were selected by identifying, term by term, the coefficients of the polynomials given in expression (29) with those of a desired Hurwitz polynomial given by $p_{o b s}(s)=\left(s^{2}+2 \zeta_{o} \omega_{n o} s+\omega_{n o}^{2}\right)^{4} \cdot\left(s+p_{o}\right)$, with $\omega_{n o}=15, \zeta_{o}=1.5$ and $p_{o}=15$. On the other hand, the controller gains, $\left\{k_{3}^{F}, \ldots, k_{0}^{F}\right\}$ and $\left\{k_{3}^{L}, \ldots, k_{0}^{L}\right\}$, governing the dominant dynamics, were set by identifying, term by term, the coefficients of the polynomials given in expression (26) with the Hurwitz polynomial $p_{\text {cont }}(s)=\left(s^{2}+2 \zeta_{c} \omega_{n c} s+\omega_{n c}^{2}\right)^{2}$, with $\omega_{n c}=2, \zeta_{c}=1$. Fig. $4 \mathrm{a}$ and Fig. 4b illustrate the path tracking and the closed loop trajectories for the coordinates of the center of mass $(x, z)$ in the earth frame showing that the system follows the desired trajectory in an accurate manner. On the other hand, Fig. 4c

(a)

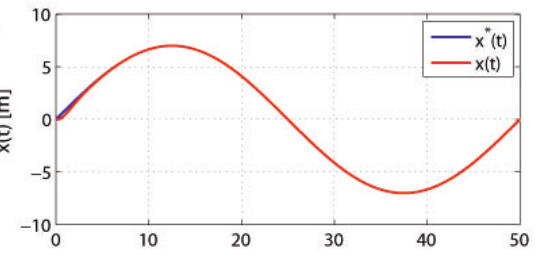

(c)

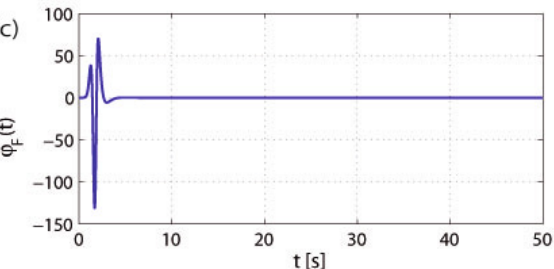

(b)
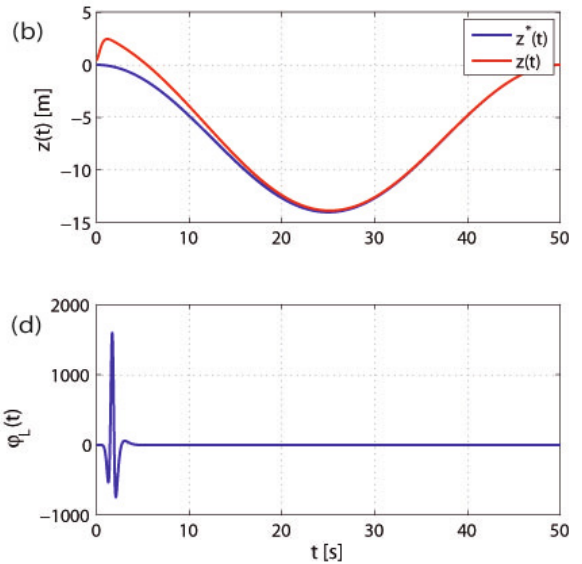

Fig. 2. Evolution of: (a) Coordinate $x$ of the center of mass in the earth frame; (b) Coordinate $z$ of the center of mass in the earth frame; (c) State-dependent estimated disturbance $\varphi_{F}$ and; (d) State-dependent estimated disturbance $\varphi_{L}$ 
and Fig. 4d depict the evolution of the GPI observer state dependent disturbance estimation.

\section{Conclusions and Future Work}

This paper is related to the introduction of biomimetic miniature air vehicles in ambient assisted living and home health applications. Indeed, the proposal described falls within the complete project "Improvement of the Elderly Quality of Life and Care through Smart Emotion Regulation". The long-term objective of the project is to find solutions for improving the quality of life and care of the elderly who can or wants to continue living at home by using emotion detection and regulation techniques. We believe that miniature air vehicles at home settings can carry some fundamental sensors to capture the mood of the ageing adult.

In this way, this particular work has explored, within the context of the trajectory tracking problem, the use of approximate, yet accurate, total active disturbance rejection schemes, based on linear GPI observers, for the flapping-wing miniature air vehicle time-averaged model. Numerical simulations were provided where the efficiency of the proposed control method is assessed. Finally, in future work, we try to extend this control scheme to the full 6 DOF flight dynamics.

Acknowledgments. This work was partially supported by Spanish Ministerio de Economía y Competitividad / FEDER under TIN2013-47074-C2-1-R grant.

1. Ellington, C.P.: The novel aerodynamics of insect flight: Applications to microair vehicles. The Journal of Experimental Biology 202, 3439-3448 (1999)

2. de Clerq, K.M.E., de Kat, R., Remes, B., van Oudheusden, B.W., Bijl, H.: Aerodynamic experiments on delfly ii: unsteady lift enhancement. In: Proceedings of the 2009 European Micro-air Vehicle Conference and Competition 2009, pp. 255-262 (2009)

3. Conn, A., Burgess, S., Hyde, R., Ling, C.S.: From natural flyers to the mechanical realization of a flapping wing micro air vehicle. In: Proceedings of the 2006 IEEE International Conference on Robotics and Biomimetics, pp. 439-444 (2006)

4. Fenelon, M.A.A.: Biomimetic flapping wing aerial vehicle. In: Proceedings of the 2008 IEEE International Conference on Robotics and Biomimetics, pp. 1053-1058 (2009)

5. Fernández-Caballero, A., Latorre, J.M., Pastor, J.M., Fernández-Sotos, A.: Improvement of the elderly quality of life and care through smart emotion regulation. In: Pecchia, L., Chen, L.L., Nugent, C., Bravo, J. (eds.) IWAAL 2014. LNCS, vol. 8868, pp. 348-355. Springer, Heidelberg (2014)

6. Castillo, J.C., Carneiro, D., Serrano-Cuerda, J., Novais, P., Fernández-Caballero, A., Neves, J.: A multi-modal approach for activity classification and fall detection. International Journal of Systems Science 45, 810-824 (2014)

7. Carneiro, D., Castillo, J.C., Novais, P., Fernández-Caballero, A., Neves, J.: Multimodal behavioral analysis for non-invasive stress detection. Expert Systems with Applications 39, 13376-13389 (2012) 
8. Oliver, M., Montero, F., Fernández-Caballero, A., González, P., Molina, J.P.: RGBD assistive technologies for acquired brain injury: description and assessment of user experience. Expert Systems (2014), doi:10.1111/exsy.12096

9. Deng, X., Schenato, L., Sastry, S.: Model identification and attitude control scheme for a micromechanical flying insect. In: International Conference on Control, Automation, Robotic and Vision, pp. 1007-1012 (2002)

10. Schenato, L., Wu, W.-C., Sastry, S.: Attitude control for a micromechanical flying insect via sensor output feedback. IEEE Transaction on Robotics and Automation 20, 93-106 (2004)

11. Campolo, D., Barbera, G., Schenato, L., Pi, L., Deng, X., Guglielmelli, E.: Attitude stabilization of a biologically inspired robotic housefly via dynamic multimodal attitude estimation. Advanced Robotics 23, 955-977 (2009)

12. Guckenheimer, J., Holmes, P.: Nonlinear Oscillations, Dynamical Systems, and Bifurcations of Vector Fields. Springer (1990)

13. Sanders, J.A., Verhulst, F.: Averaging Methods in Non-Linear Dynamical Systems. Springer (1985)

14. Deng, X., Schenato, L., Wu, W.-C., Sastry, S.: Flapping flight for biomimetic robotic insects: part II-flight control design. IEEE Transactions on Robotics 22, 789-803 (2006)

15. Rifaï, H., Marchand, N., Poulin-Vittrant, G.: Bounded control of an underactuated biomimetic aerial vehicle-Validation with robustness tests. Robotics and $\mathrm{Au}-$ tonomous Systems 60, 1165-1178 (2012)

16. Khan, Z.A., Agrawal, S.K.: Control of longitudinal flight dynamics of a flappingwing micro air vehicle using time-averaged model and differential flatness based controller. In: Proceedings of the 2007 American Control Conference, pp. 5284-5289 (2007)

17. Han, J.: From PID to active disturbance rejection control. IEEE Transactions on Industrial Electronics 56, 900-906 (2009)

18. Radke, A., Gao, Z.: A survey of state and disturbance observers for practicioners. In: Proceedings of the 2006 American Control Conference, pp. 5183-5188 (2006)

19. Qing, Z., Gao, Z.: On practical applications of active disturbance rejection control. In: Proceedings of the 29th Chinese Control Conference, pp. 6095-6100 (2010)

20. Luviano-Juárez, A., Cortés-Romero, J., Sira-Ramírez, H.: Synchronization of chaotic oscillators by means of generalized proportional integral observers. International Journal of Bifurcation and Chaos 20, 1509-1517 (2010)

21. Fliess, M., Lévine, J., Martin, P., Rouchon, P.: Flatness and defect of nonlinear systems: Introductory theory and examples. International Journal of Control 61, 13271361 (1995)

22. Sira-Ramírez, H., Agrawal, S.: Differentially Flat Systems. Marcel Dekkert Inc. (2004)

23. Sira-Ramírez, H., Núñez, C., Visairo, N.: Robust sigma-delta generalized proportional integral observer based on a 'buck' converter with uncertain loads. International Journal of Control 83, 1631-1640 (2009)

24. Morales, R., Sira-Ramírez, H., Somolinos, J.A.: Robust control of underactuated wheeled mobile manupulators using GPI disturbance observers. Multibody Systems Dynamics 32, 511-533 (2014)

25. Morales, R., Sira-Ramírez, H., Somolinos, J.A.: Linear active disturbance rejection control of the hovercraft vessel model. Ocean Engineering 96, 100-108 (2015) 